\title{
Experimental Photon Doubling as a Possible Local Inference of the Hubble Parameter
}

\author{
Stanley A. Koren, Blake T. Dotta and Michael A. Persinger*
}

Laurentian University, Sudbury, Ontario, P3E 2C6, Canada

\begin{abstract}
Mach's principle of immanence of the universe states that the properties of local matter depend upon the presence of the remainder of the universe. The discrete solution for the intrinsic duration of any space is the product of Hubble's expansion parameter and the length of the space divided by $\sqrt{\hbar \mathrm{Gc}^{-3}}$. For the typical "space" occupied by protons and electrons these values are $\sim 3 \mathrm{~ms}$ and $\sim 1 \mathrm{~ms}$, respectively. Recently we demonstrated that the durations of photon emissions from chemiluminescent reactions doubled during simultaneous local+nonlocal activations if both loci shared a specific pattern of changing angular velocities of $1 \mathrm{~ms}$ point-duration configurations of magnetic fields within circular arrays. In the present experiments we varied the point durations in integers between 1 and $10 \mathrm{~ms}$ and found only the predicted 3 $\mathrm{ms}$ and $1 \mathrm{~ms}$ point durations produced this effect. On the bases of these earth-based measures Hubble's constant would be $\sim 74.8 \mathrm{~km} \cdot \mathrm{s}^{-1} \cdot \mathrm{MParsec}^{-1}$. The results suggest an easier and more economic inference of this important cosmological parameter and alternative applications to protons and electrons during chemical reactions.
\end{abstract}

Keywords: Changing angular velocity circular magnetic fields, chemiluminescence, electron diameter, entanglement, hubble's parameter, proton.

\section{INTRODUCTION}

The principle of immanence of the universe developed by the late $19^{\text {th }}$ century physicist Ernst Mach indicated that the properties of local matter depend on the presence of the remainder of the universe. Stated alternatively the behavior of any portion of the universe is determined by all of its parts [1]. The corollary to Mach's principle indicates that the natural laws governing the largest scale of space-time should be intrinsically related to the laws governing the smallest scale of space-time. Although experimental support for this interesting assumption has often eluded empirical verification we report here an experiment that may support the basic proposition.

Expansion of the universe is inferred primarily by Hubble's constant whose estimates have ranged between 50 and $100 \mathrm{~km} \cdot \mathrm{s}^{-1} \cdot \mathrm{MParsec}^{-1}$. When Hubble's expansion parameter $\left(\mathrm{H} \sim 10^{-18} \mathrm{~s}^{-1}\right)$ is multiplied by a target distance and this velocity is divided by Planck's length $\left[(\mathrm{H} \cdot \mathrm{d}) \cdot \mathrm{P}_{\mathrm{L}}{ }^{-1}\right]$, a duration is obtained. Assuming a median value of $75 \mathrm{~km} \cdot \mathrm{s}^{-1} \cdot \mathrm{MParsec}^{-1}$ the temporal increments for the standard "wavelengths" or diameters of a proton or an electron are $3 \mathrm{~ms}$ and $1 \mathrm{~ms}$, respectively. We $[2,3]$ interpreted these values as the time required for the space occupied by the target length, the proton or electron, to expand one Planck's length. We have been devising an experimental strategy to test this interpretation.

If Mach's contentions were correct, then his principle should be evident at macro- as well as micro-levels of space

*Address correspondence to this author at the Laurentian University, Sudbury, Ontario, P3E 2C6, Canada; Tel: 01-705-675-4824;

Fax: 01-705-671-3844; E-mail: mpersinger@laurentian.ca and matter. This is relevant to astronomers and physical cosmologists, particularly if even a subset of Eddington's approach to conceptualizing the universe has application [4]. The inspiration for this research began when pairs of human subjects displayed excess correlations when separated by distance $(\sim 10 \mathrm{~m})$ if they shared the same circular, angularly accelerating magnetic fields around their heads. A schematic of this experimental paradigm is shown in Fig. (1). Presentation of light flashes to one person (e.g., "local stimulus) sitting in one room resulted in the emissions of photons as measured by a photomultiplier tube (PMT) from the head of the other person (e.g., "non-local response") sitting blindfolded in another darkened room [5]. The increased output was in the order of $10^{-11} \mathrm{~W} \cdot \mathrm{m}^{-2}$.

This particular quantity for radiant power density was salient from a physical chemistry perspective of brain matter. It was equivalent to the individual energy of $\sim 2 \cdot 10^{-20} \mathrm{~J}[6]$ per action potential from approximately $10^{7}$ neurons each generating 10 action potentials, which is approximately the numbers of neurons associated with discrete cognitive processes [5]. This "excess correlation" only occurred when the light was flashed to the person in the other room and specific rotational parameters of the fields were in operation for both participants. Stated alternatively the effect was evident when the rotation of the magnetic fields displayed increasing followed by decreasing angular velocities around the heads of the two participants separated by non-traditional distances. A similar effect was associated with light flashes to cell cultures containing $\sim 10^{6}$ melanoma cells. During the same rotational parameters, light flashes to one dish was associated with light emissions from the $\sim 10^{6}$ cells in the other dish housed in another room in the dark as long as both dishes shared the specific magnetic fields. The estimated energy 


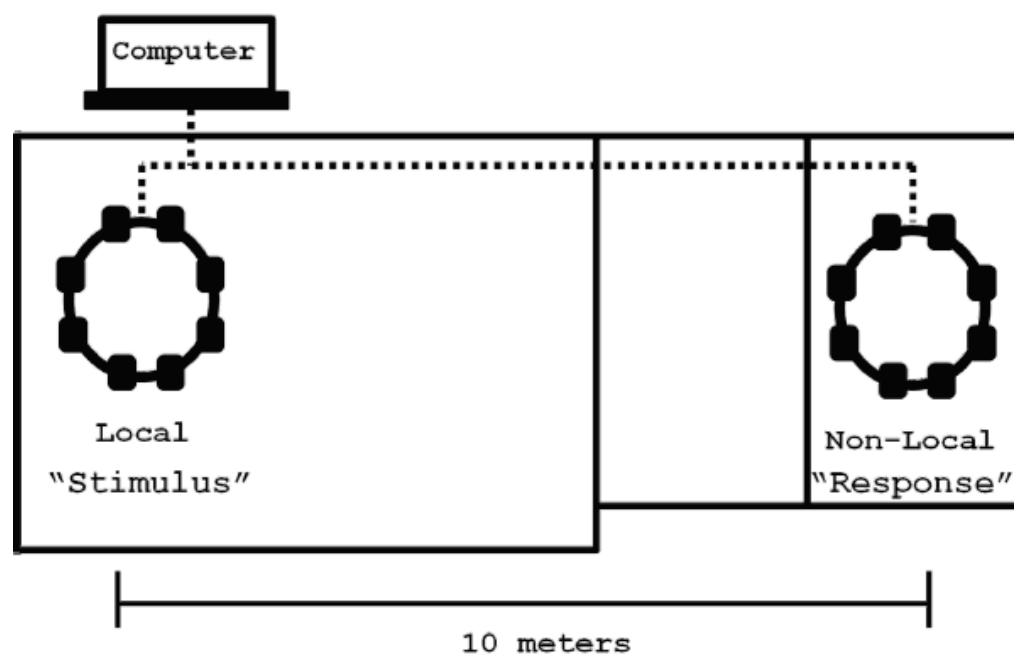

Fig. (1). Diagram of procedure by which the same magnetic field condition was generated in two separate spaces separated by about $10 \mathrm{~m}$. The two circular arrays represent the 8 solenoids. The magnetic fields were generated as brief pulses whose durations either increased or decreased as the programmable fields were rotated around the solenoids in a counterclockwise direction. The pattern of the magnetic field that was generated reflected either a decreasing or increasing frequency or "phase" modulation. See [7] for more details of field patterns.

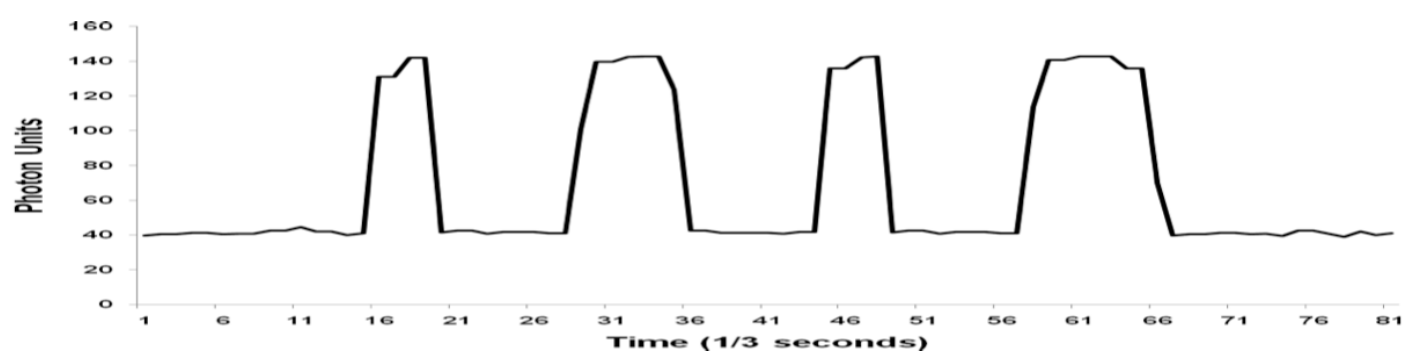

Fig. (2). Examples of durations of photon emission pulses from local (thin, 1 and 3) and nonlocal+local (wider, 2 and 4 ) reactant injections during optimal experimental conditions.

\section{AD Configuration}

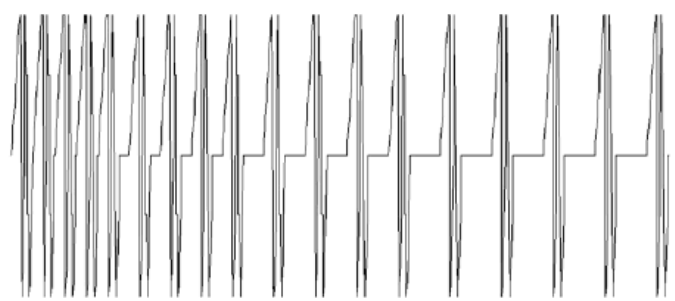

\section{Configuration}

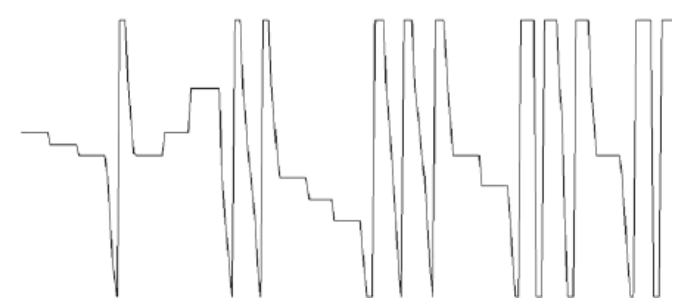

Fig. (3). Actual shapes of the decreasing frequency modulated field (AD) embedded within the increasing angular group velocity condition and the increasing frequency field (DI) embedded within the decreasing angular velocity condition.

associated per cell associated with these emissions was calculated to be about $10^{-20} \mathrm{~J} \cdot \mathrm{s}^{-1}[6]$.

To insure the effect was not dependent upon the complexity of living systems, Dotta and Persinger [7] injected $0.1 \mathrm{cc}$ of hydrogen peroxide into a solution of $3 \%$ sodium hypochlorite to produce a robust chemilumiscent reaction that was detected by the PMT sensor positioned immediately below the plate and was visually conspicuous.

The chemically-induced photon emission was about 100 fold greater than the background levels $\left(5 \cdot 10^{-11} \mathrm{~W} \cdot \mathrm{m}^{-2}\right)$ with durations of about $2 \mathrm{~s}$. When both experimenters each injected $0.1 \mathrm{cc}$ of peroxide simultaneously into two different dishes of hypochlorite separated by $10 \mathrm{~m}$, there was a "doubling" of the photon emission (Fig. 2) that was not only ob- vious (that is, not requiring statistical extraction to be discernable) but comparable to a single injection of $0.2 \mathrm{cc}$ of peroxide into one plate only. The effect was clearly evident even when the two loci were separated by $\sim 3 \mathrm{~km}$, the longest distance tested to date.

Multiple experiments demonstrated that this doubling effect, as if the two separate distal locations occupied the same space, only occurred when both plates were positioned in the center of rotating magnetic fields with decelerating group velocities embedded with an increasing frequency modulation pattern after they were first exposed to accelerating group velocities containing a decreasing frequency modulation pattern (Fig. 3). Other combinations of the envelope and the moving wave pattern were not effective (Fig. 4). Sym- 


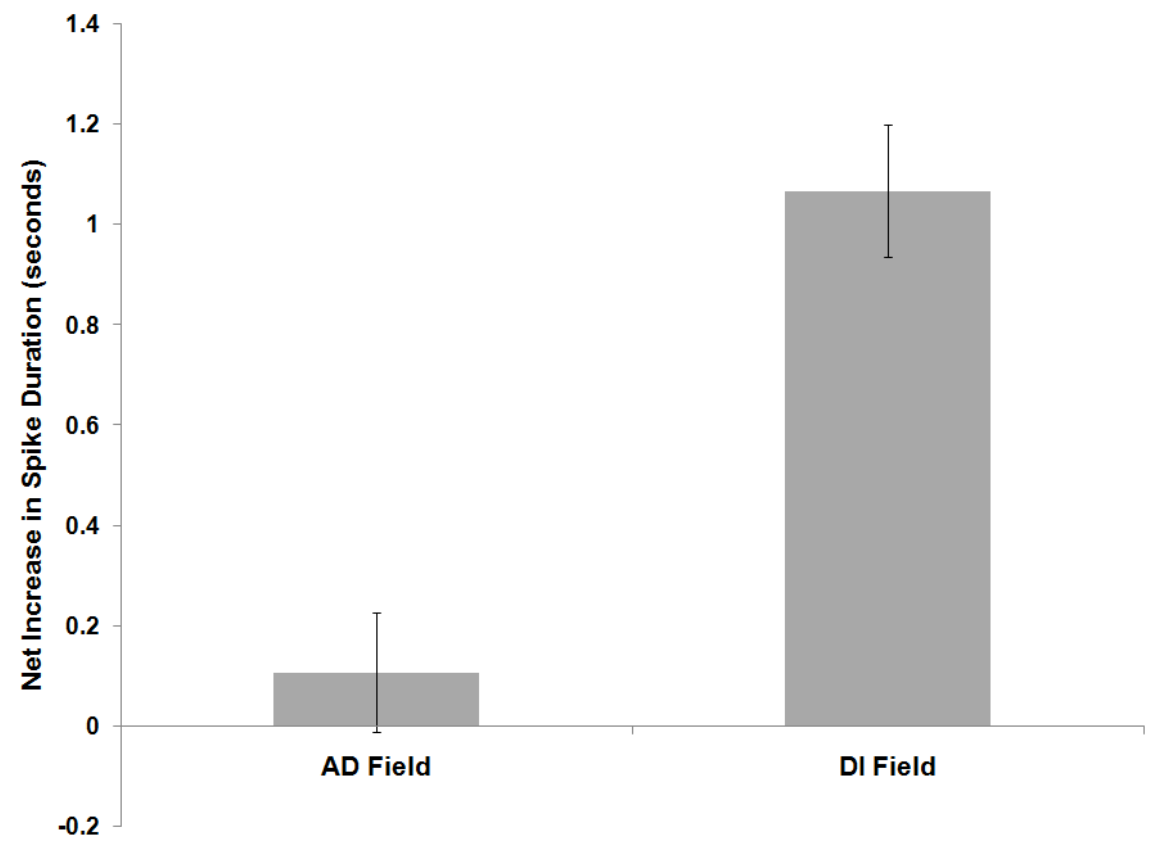

Fig. (4). Net increases in the photon spike durations during nonlocal+local simultaneous injections during the optimal procedure (DI field presentation).

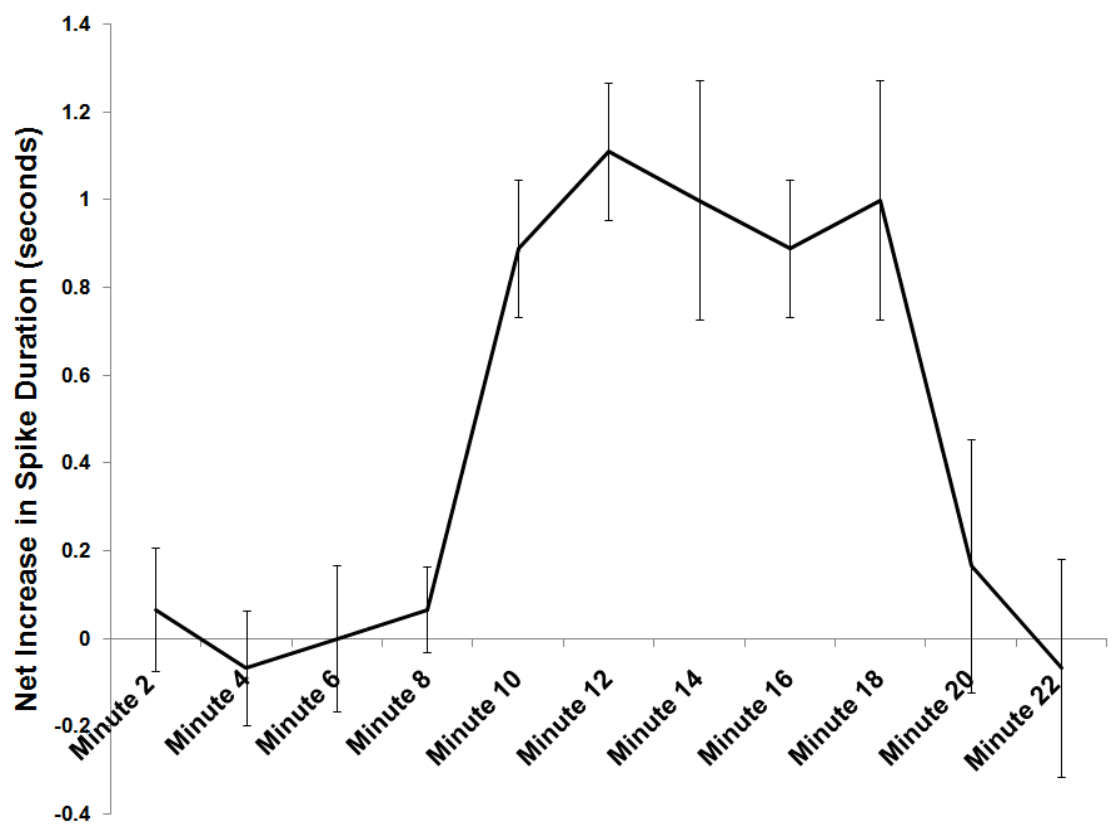

Fig. (5). Durations of the AD (accelerating group velocity embedded within a decreasing angular velocity) condition required to produce the "double photon" effect during the DI condition. Vertical bars indicate \pm 1 standard deviations.

metrical patterns such as sinusoidal $7 \mathrm{~Hz}$ fields were also not effective.

The duration of this doubling effect during simultaneous nonlocal+local injections was limited to about $8 \mathrm{~min}$ before subsequent paired injections produced effects that were not different from single injections (Fig. 5). There was also a non-linear relationship [6] between the duration of the antecedent condition (group velocity accelerating and embedded wave form with slowing frequency modulation) and the occurrence of the effect. In our very reliable experiments completed over the duration of a year, the point duration was always $1 \mathrm{~ms}$.
In the present experiments reported here we examined systematically each integer point duration from $1 \mathrm{~ms}$ to 10 $\mathrm{ms}$. According to $(\mathrm{H} \cdot \mathrm{d}) \cdot \mathrm{P}_{\mathrm{L}}^{-1}$ there should be two peaks in the occurrence of the "photon doubling", $1 \mathrm{~ms}$ associated with the classical electron diameter and $3 \mathrm{~ms}$ associated with the proton wavelength. Because the chemiluminecent reactions occurred in water traditional interpretations indicate that both protons and electrons would be more or less equally involved. We predicted the photon emissions during the nonlocal+local injections of reactant should display the "doubling" with either $1 \mathrm{~ms}$ or $3 \mathrm{~ms}$ point durations while for all other point durations (particularly $2 \mathrm{~ms}$ ) the local+nonlocal responses should not differ from those of local injections. 


\section{METHODS AND MATERIALS}

The paradigm in the present study is shown in Fig. (1) and was the same equipment utilized in the human brain and cell studies [5]. The details of our exposure procedures have been published elsewhere [7]. Essentially, two identical arrangements of 8 pairs of solenoids arranged in a circle $(\sim 60$ $\mathrm{cm}$ circumference) were placed in two separate rooms separated by $\sim 10 \mathrm{~m}$. Each of the 8 pairs of solenoids were housed in plastic canisters and distributed equally ( $45 \mathrm{deg}$ ) around the circle. The plates, each containing $6 \mathrm{cc}$ of the hypochlorite solutions, were placed in the center of each ring of solenoids. For the plate that was placed above the PMT sensor which was surrounded by one of the circular arrays of solenoids but covered with several layers of thick cloth to minimize light interference, the hydrogen peroxide was delivered in $0.1 \mathrm{cc}$ quantities from a $1 \mathrm{cc}$ syringe through intramedic tubing. For the other plate in the other room (which was also an acoustic-chamber and Faraday cage) the $0.1 \mathrm{cc}$ of hydrogen peroxide was injected from a $1 \mathrm{cc}$ syringe.

We followed the optimal protocol that produced the "photon doubling" effect in the previous studies. During the first 6 min both plates were exposed continuously to the accelerating group velocity embedded with a decreasing frequency modulation field. The acceleration was produced by decreasing the field duration (AD configuration) at each solenoid by $2 \mathrm{~ms}$ from the base duration of $20 \mathrm{~ms}$. Hence the duration of the field at the last solenoid was $6 \mathrm{~ms}$, before it began again at $20 \mathrm{~ms}$. On the other hand the decreasing group velocity contained the increasing frequency modulated pattern (DI configuration) and was created by adding $2 \mathrm{~ms}$ at each solenoid from the base duration of $20 \mathrm{~ms}$. As a result, the duration of the field at the $8^{\text {th }}$ solenoid was $34 \mathrm{~ms}$. The fields were generated from custom constructed DOS-based programmable software that converted a column of numbers between 0 and 256 to voltages between $-5 \mathrm{~V}$ and $+5 \mathrm{~V}$ $(127=0 \mathrm{~V})$ that were controlled by the same IBM $286 \mathrm{com}$ puter. (In our distal experiments, at $3 \mathrm{~km}$, the configurations were controlled by two different IBM 286 computers whose initiation was synchronized by electronic stop watches).

The discrete voltages were transmitted to customconstructed digital-to-analogue converters before being delivered to each pair of solenoids within the circular array in each room. The duration of the field at each set of solenoids and the rate of change, e.g., +2 or $-2 \mathrm{~ms}$ was also controlled by the software and by circuitry described in U.S. Patent 6,312,376 B1: Nov. 6, 2001 and Canadian Patent No. 2213296. During the subsequent $18 \mathrm{~min}$ after the initiation of the first phase $0.1 \mathrm{cc}$ of hydrogen peroxide was injected into the dish over the PMT sensor at the beginning of every odd minute while $0.1 \mathrm{cc}$ was injected into each of the two dishes simultaneously every even minute by two different experimenters. The synchronization was insured by coupled digital stop watches on each experimenter's cell phone. The duration of each injection was practiced to $2 \mathrm{~s}( \pm 0.1 \mathrm{~s})$. The area under the curve for each single and "double injection" event was calculated from the digital data from the IBM laptop that was employed to measure the photon emissions through a voltmeter. This was completed by summing the number of units that deviated by more than 10 units above the background baseline before the return to baseline. These values were correlated $>0.90$ with the calculated area under the curve of the spike and were selected for convenience.

We applied our standard protocol which produced the maximum "photon doubling" effect for the $1 \mathrm{msec}$ point durations. This protocol involved local injections 1,3 , and 5 min and local+nonlocal injections at 2, 4, and 6 min after the beginning of the exposures to the accelerating group velocity embedded with decreasing frequency modulated field. The decelerating group velocity embedded with an increasing frequency modulated field was then begun and continued for the next $10 \mathrm{~min}$. Local injections were given at 9, 11, 13, 15 and $17 \mathrm{~min}$ while the nonlocal+local (simultaneous) injections were given at 10,12,14, 16 and $18 \mathrm{~min}$. Previous experiments indicated that "photon doubling" effect was evident at $10 \mathrm{~min}$ and was no longer present after $\sim 18 \mathrm{~min}$, i.e., a net duration of $\sim 8 \min [7]$.

The means and standard deviations for the net differences between the durations of the photon spikes for nonlocal+local injections subtracted from the local only injections were obtained for the 3 pairs of responses during the first 6 minutes (accelerating group velocity, decreasing frequency modulation circular magnetic fields) and for the 5 pairs of local and nonlocal+local injections for the subsequent 10 to $18 \mathrm{~min}$ (decelerating group velocity, increasing frequency modulation circular magnetic fields). We completed triplicates for the 1,2, and $3 \mathrm{~ms}$ point durations to insure reliability (15 trials for each point duration for the decelerating group velocity trials) and one replicate (5 trials) for the 4 through $10 \mathrm{~ms}$ point durations because there was no evidence of any effect for any of them. The " 0 " point duration, that is the port time of the computer ( $200 \mu \mathrm{s})$, was also included.

There were a total of 18 experimenters on 18 different days over a two month period. The magnetic field strengths, as measured by a standard power meter, registered a peak of $1.9 \mu \mathrm{T}$ in the center of the rings of solenoids for all point durations. The average field strengths ranged between 1.0 and $1.2 \mu \mathrm{T}$ for all point durations.

\section{RESULTS AND DISCUSSION}

The results were clear and conspicuous as shown in Fig. (6). The only point durations that were associated clearly with the "photon doubling" effect were $1 \mathrm{~ms}$ and $3 \mathrm{~ms}$. The absence of any robust effect with the $2 \mathrm{~ms}$ duration supports the interpretation that the phenomena are discrete and not artifacts of subtle changes in field strengths associated with the different durations. In fact there was no appreciable difference in averaged field strengths for the different point durations.

If $3 \mathrm{~ms}$ durations are associated with the expansion of the proton length by one Planck's length, then effects upon membranes which are strongly dependent upon correlative proton channels and hydronium ions [8] within water might be expected. On the other hand for the present study, both protons (in the water) and electrons (orbital "shell" shifts) would have both been involved with the chemoluminescent reaction. The source of the photon emissions from the $\mathrm{H}_{2} \mathrm{O}_{2}$ $+\mathrm{NaClO}$, resulting in $\mathrm{H}_{2} \mathrm{O}+\mathrm{NaCl}+\mathrm{O}_{2}$ or more precisely $\mathrm{HOOH}+\mathrm{OCl}^{-} \rightarrow{ }^{1} \mathrm{O}_{2}$ is the excited singlet oxygen. 


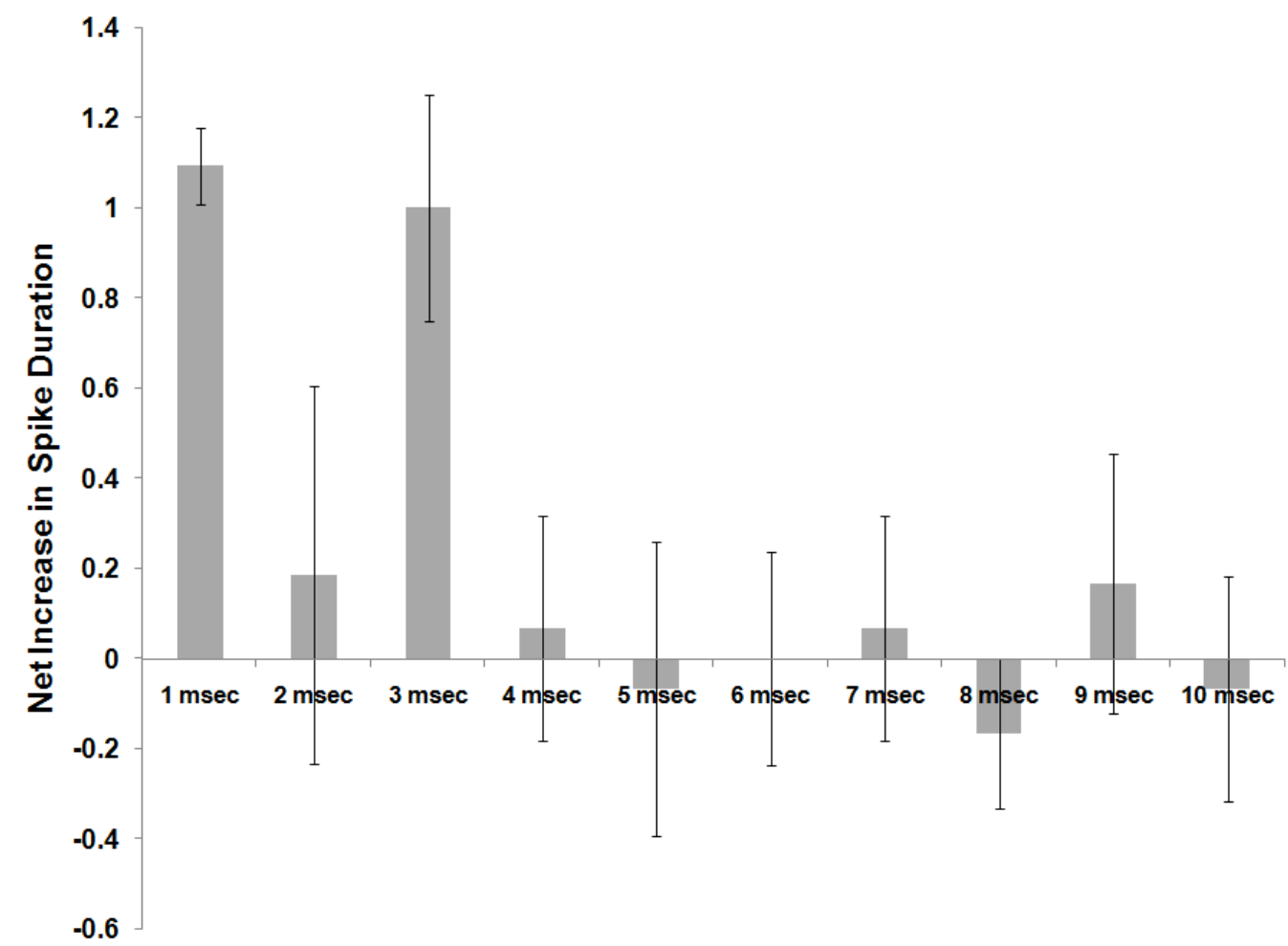

Fig. (6). Net increases in photon emissions during the nonlocal+local injections (the "photon doubling effect") as a function of the point duration of each value that generated the magnetic field sequence. Vertical bars indicate \pm 1 standard deviations.

We suggest that this model may be useful for pursuing the non-intuitive solutions for the boundaries of $\left[(\mathrm{H} \cdot \mathrm{d}) \cdot \mathrm{P}_{\mathrm{L}}^{-1}\right]$. If the estimated width of the universe $\left(10^{26} \mathrm{~m}\right.$ or inverse

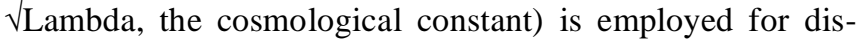
tance, then the time required for the space of the entire universe to expand one Planck's length would be $\sim$ Planck's time or the base frequency of the Zwitterbewegung, that is, the equivalent of one period of the zero point fluctuation [9]. If Planck's length was employed as the distance, then the time required to expand one Planck's length would be about $10^{17} \mathrm{~s}$, which is within the order of magnitude of the age of the universe. In other words the time required for the universe to jump one Planck's length is Planck's time while the time required for one Planck's length to jump one Planck's length is the age of the universe [2]. Such apparently counterintuitive results at the boundary for maximum and minimum values may be required to accommodate some Casimir solutions [3] and holographic considerations.

By far the most important practical application of this result is the possibility for a local inference of measurement for Hubble's constant which has been employed in many models. In the past its value has ranged between 50 and 100 $\mathrm{km} \cdot \mathrm{s}^{-1} \cdot \mathrm{MParsec}^{-1}$ with the equivalent parameters between 1.6 and $3.2 \cdot 10^{-18} \mathrm{~s}^{-1}$. Our present software permitted only integers to determine the point durations of the frequencymodulated (and crudely phase-modulated) magnetic fields within the envelopes (group velocities) that were rotated with increasing or decreasing angular velocities around the reactions. If we assumed these integers were the most accurate then the solution for both proton and electron parameters would indicate Hubble's constant would be $\sim 74.8 \mathrm{~km} \cdot \mathrm{s}^{-}$ ${ }^{1} \cdot \mathrm{MParsec}^{-1}$. Programming of intermittent values for point durations and more rapid sampling of the photon emissions might allow more precise estimations of Hubble's parameter locally as well as its potential fluctuations over real time. If the principle upon which these observations is valid, the implications for the foundations of physics could be profound. Because the equipment is easy to construct and the experiments are quickly executed replication by other laboratories will be the ultimate test of the degree to which our results here generalize to other locations.

\section{CONCLUSION}

Mach's principle of immanence of the universe indicates that the properties of local matter should reflect the entire universe. Recent measurements of conspicuous increases in the duration of photon emissions from electromagneticallyyoked chemical reactions separated by a significant distance when both loci share specific patterns of angularly accelerating magnetic fields may be a support of this proposition. The specific point durations for the optimal photon enhancing effect were the same values that were predicted from the theoretical expansion of a proton or electron according to the Hubble parameter. We suggest that this local effect reflects the universal laws that also govern much larger space.

\section{CONFLICT OF INTEREST}

The authors confirm that this article content has no conflicts of interest.

\section{ACKNOWLEDGEMENTS}

We thank Dr. Ed. Bosarge, Jr., CEO of Capital Technologies, Inc. for financial support. 


\section{REFERENCES}

[1] Singh G. Great ideas and theories in modern cosmology. New York: Dover 1961.

[2] Persinger MA, Koren SA. A theory of neurophysics and quantum neuroscience: implications for human brain function and the limits of consciousness. Int J Neurosci 2007; 117: 157-75.

[3] Koren SA, Persinger MA. The Casimir force along the universal boundary: quantitative solutions and implications. J Astrophys Phys Phys Cosmol 2010; 4: 1-4.

[4] Persinger MA. Support for Eddington's numbers and his approach to astronomy: recent developments in the physics and chemistry of the human brain. Int Lett Chem Phys Astron 2013; 8: 8-19.

[5] Dotta BT, Buckner CA, Lafrenie RM, Persinger MA. Photon emissions from human brain and cell cultures exposed to distally rotat- ing magnetic fields shared by separate light-stimulated brains and cells. Brain Res 2011; 338: 77-88.

[6] Persinger MA. $10^{-20}$ Joules as a neuromolecular quantum in medicinal chemistry: an alternative approach to myriad molecular pathways? Curr Med Chem 2010; 17: 3094-8.

[7] Dotta BT, Persinger MA. "Doubling of local photon emissions when two simultaneous spatially-separated reactions share the same magnetic field configurations. J Biophys Chem 2012; 3: 72 80 .

[8] DeCoursey TE. Voltage-gated proton channels and other proton transfer pathways. Physiol Rev 2003; 83: 475-9.

[9] Puthoff HE. Gravity as a zero point-fluctuation force. Phys Rev A 1989; 39: 2333-42.

Received: October 16, 2013

(C) Koren et al.; Licensee Bentham Open.

This is an open access article licensed under the terms of the Creative Commons Attribution Non-Commercial License (http://creativecommons.org/licenses/by-nc/3.0/) which permits unrestricted, non-commercial use, distribution and reproduction in any medium, provided the work is properly cited. 